Check for updates

Cite this: RSC Adv., 2021, 11, 21695

Received 31st May 2021

Accepted 14th June 2021

DOI: 10.1039/d1ra04214a

rsc.li/rsc-advances

\section{Synthesis of new allylidene amino phenol- containing Schiff bases and metal complex formation using trimethinium salts $\dagger$}

\author{
Ziba Rafiee Samani and Abdolmohammad Mehranpour (DD* \\ An efficient route for the synthesis of novel Schiff bases from the condensation reaction of 2-substituted \\ 1,3-bis(dimethylamino)-trimethinium salts with diverse aminophenols in the presence of triethylamine in \\ $\mathrm{EtOH}$ at reflux is described. Complexes of transition metals with Schiff base ligand (L) 3c, having the \\ donor atom set $\mathrm{N}_{2} \mathrm{O}_{2}$, were studied. The ultraviolet spectral behavior of the complexes in DMSO was \\ investigated and the $\lambda_{\max }$ of these compounds was examined. The structure of the new compounds was \\ confirmed based on their spectral data from IR, ${ }^{1} \mathrm{H}$ NMR and ${ }^{13} \mathrm{C} N M R$, mass spectra, and elemental analysis.
}

\section{Introduction}

Schiff bases are some organic compounds that are highly used. They are used as pigments and dyes, intermediates in organic synthesis, catalysts, and polymer stabilizers. Schiff bases exhibit a wide range of biological activities, including antifungal, antiviral, antimalarial, antibacterial, anti-inflammatory, antiproliferative, and antipyretic properties. ${ }^{\mathbf{1 - 6}}$

Schiff base ligands have the capability of coordinating metals through imine nitrogen and another group. Nowadays active and well-designed Schiff base ligands are considered "privileged ligands". Schiff bases are able to stabilize many different metals in various oxidation states, controlling the performance of metals in a wide variety of useful catalytic transformations. ${ }^{7-9}$

Metal complexes of Schiff bases play a central role in the development of coordination chemistry. This situation is manifested by the huge number of publications ranging from purely synthetic to modern physicochemical to biochemically relevant studies of these complexes. A wide variety of stable chemical species have been synthesized containing both transition and nontransition metals and multifarious ligand systems. ${ }^{10,11}$

The vinamidines, with saturated nitrogen as the $\mathrm{p}$-donor and the imino group as the $\pi$-acceptor, are of particular interest to us. Vinamidinium salts have long found practical use as versatile three-carbon building blocks in the synthesis of heterocyclic benzenoid and nonbenzenoid aromatic rings, from cyclic and acyclic precursors alike. One of the useful attributes of many vinamidinium salts is their ease of preparation from

Department of Chemistry, Faculty of Sciences, Persian Gulf University, Bushehr, 75169, Iran.E-mail: ammehranpour@hotmail.com

$\dagger$ Electronic supplementary information (ESI) available. See DOI: 10.1039/d1ra04214a substituted acetic acids under Vilsmeier-Haack conditions. ${ }^{12-23}$ "In this study, our research group was able to prepare $\beta$ substituted trimethinium salts with different $\mathrm{R}$ groups (including aryls and heteroaryls) from correspondingly substituted acetic acids, $\mathrm{R}-\mathrm{CH}_{2} \mathrm{CO}_{2} \mathrm{H}$, with good yields". ${ }^{24,25}$

In continuation of this research on applications of trimethinium salts in organic synthesis (Scheme 1), ${ }^{26-31}$ in this study, we report a new and highly efficient method for the synthesis of a novel class of allylidene amino phenol-containing Schiff bases from the reaction of trimethinium salts and diverse aminophenols in the presence of triethylamine, under catalyst-free conditions in EtOH at reflux (Scheme 2).

\section{Results and discussion}

Eight new Schiff base derivatives with the general formula $\mathrm{N}_{2} \mathrm{O}_{2}$, were synthesized from the reaction of different aminophenols

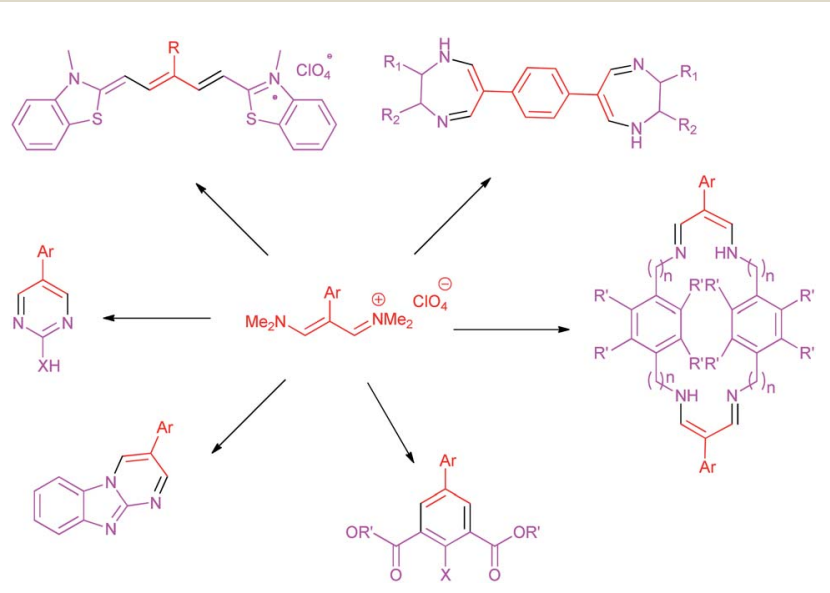

Scheme 1 Application of trimethinium salts in synthetic organic chemistry. 

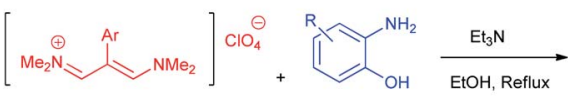

2

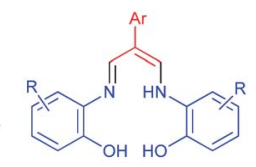

3
Scheme 2 Synthesis of Schiff bases 3 via the reaction between trimethinium salts 1 and diverse aminophenols 2 in the presence of $\mathrm{Et}_{3} \mathrm{~N}$ in $\mathrm{EtOH}$ at reflux.

and various trimethinium salts in the presence of triethylamine in ethanol as a solvent at reflux. Initially, $\mathrm{N}$-(2-(4-bromophenyl)-3(dimethylamino)-allylidene)- $N$-methylmethanaminium perchlorate (1e) and 2-amino-4-chlorophenol (2b) were chosen as the model substrates to optimize the reaction conditions, (the reason for this choice is the good TLC of these two reactants for observations), such as various reagent sources and solvents. The results are summarized in Table 1 . In this study, various reagents such as $\mathrm{NaH}$ and $\mathrm{NaOCH}_{3}$ were examined and it became clear that they cannot continue this reaction (Table 1, entries 1 and 2). The bases such as $\mathrm{Et}_{3} \mathrm{~N}$ and $\mathrm{i}-\mathrm{Pr}_{2} \mathrm{NEt}$ were examined in $\mathrm{EtOH}$, in which resulted higher yield and shorter reaction time when the reaction was carried out in the presence of (1 eq.) of the $\mathrm{Et}_{3} \mathrm{~N}$ (Table 1, entries 3 and 4). The effect of solvents were also investigated and it was observed that the desired product was not obtained in the solvents $\mathrm{CH}_{3} \mathrm{CN}$ and DMF. However, the reaction was obtained highly effective with solvents such as EtOH and $\mathrm{MeOH}$ (Table 1, entries 4-7). The control experiment confirmed that the reaction has not occur in the absence of the base and acid conditions (Table 1, entries 8 and 9).

In the next stage, the efficiency of the process under optimized conditions was explored. For this purpose, trimethinium salts 1a-f were condensed with aminophenol derivatives $\mathbf{2 a - c}$ in

Table 1 Optimization of the reaction conditions ${ }^{a}$

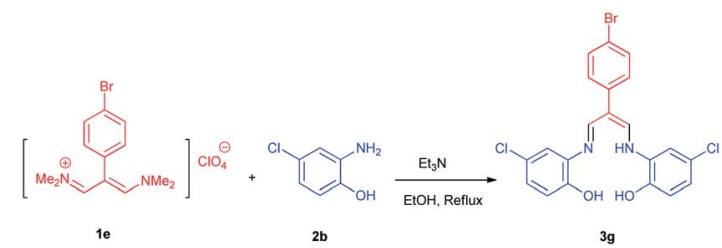

\begin{tabular}{lllll}
\hline Entry & Conditions & Solvent & Time (h) & Yield $^{b}(\%)$ \\
\hline 1 & $\mathrm{NaH}$ & EtOH & 24 & - \\
2 & $\mathrm{NaOCH}_{3}$ & $\mathrm{EtOH}$ & 24 & - \\
3 & $\mathrm{i}-\mathrm{Pr}_{2} \mathrm{NEt}$ & $\mathrm{EtOH}$ & 20 & 55 \\
4 & $\mathrm{Et}_{3} \mathrm{~N}$ & $\mathrm{EtOH}$ & 12 & 90 \\
5 & $\mathrm{Et}_{3} \mathrm{~N}$ & $\mathrm{MeOH}$ & 12 & 70 \\
6 & $\mathrm{Et}_{3} \mathrm{~N}$ & $\mathrm{CH}_{3} \mathrm{CN}$ & 24 & - \\
7 & $\mathrm{Et}_{3} \mathrm{~N}$ & $\mathrm{DMF}$ & 24 & - \\
8 & - & EtOH & 24 & - \\
9 & $\mathrm{AcOH}$ & $\mathrm{EtOH}$ & 24 & -
\end{tabular}

${ }^{a}$ Reaction conditions: $\quad N$-(2-(4-bromophenyl)-3-(dimethylamino)allylidene)- $N$-methylmethanaminium perchlorate 1e $(1 \mathrm{mmol}), 2$ amino-4-chlorophenol $2 \mathbf{b}$ ( $2 \mathrm{mmol})$, base (1 eq.), solvent $(15 \mathrm{~mL})$, 12 h. ${ }^{b}$ Isolated yield. the presence of $\mathrm{Et}_{3} \mathrm{~N}$ ( 1 eq.) to afford the corresponding products $3 \mathbf{3}-\mathbf{i}$ in high yields.

The synthetic pathway to synthesis the titled compounds is consisting of two steps. At first, compounds 1 were prepared similar to the previous studies. ${ }^{18-25}$ Then the results were treated with aminophenol derivatives to afford the related Schiff base ligands as the desired products. As Table 2 indicates, a variety of trimethinium salts were successfully applied in this process to afford the corresponding Schiff base ligands derivatives as novel compounds with excellent yields.

As shown in Table 2, compound 3a unlike the other compounds, creates the 1,4-oxazpine salt as a product, probably due to the lack of electrons of the unsubstituted pyridine ring, which causes an intramolecular reaction. In the other molecules, the electron deficiency is less and there is enough time to perform the intermolecular reaction.

\section{The complexation steps}

The complexation of a series of new allylidene amino ortho hydroxy-containing Schiff bases as ligand with different metal ions was studied by means of the ultraviolet-visible spectrophotometry technique in DMSO as a solvent. Copper, zinc, cobalt, and nickel Schiff bases were prepared using the corresponding acetate $\mathrm{M}(\mathrm{OAc})_{2}(\mathrm{M} \sim \mathrm{Cu}, \mathrm{Zn}, \mathrm{Co}, \mathrm{Ni})$. For this purpose, compound 3c was chosen; then, UV-Vis absorption spectrum of the ligand $3 \mathbf{c}$ and its complexes were investigated in range of $190-840 \mathrm{~nm}$ in DMSO solvent (Scheme 3). The ultraviolet-visible electronic spectrum of ligand $3 c$ shows the absorption peak at (370) $\mathrm{nm}$, which can be attributed to $\mathrm{n} \rightarrow \pi^{*}$ electronic transitions. As shown in Scheme 3, for all complexes, maximum absorption wavelengths shifted to longer values (bathochromic effect) compared to the ligand $3 \mathbf{c}$ indicating the complex formation.

The proposed mechanism for the formation of Schiff bases $3 \mathbf{b}-\mathbf{i}$ in the presence of $\mathrm{Et}_{3} \mathrm{~N}$ is shown in Scheme 4. First, intermediate $\mathbf{A}$ is formed by the nucleophilic attack of the amine group in aminophenol to trimethinium salt 1. Then, removal of dimethylamine occurs, followed by the nucleophilic attack of the second molecule of aminophenol on the obtained iminium salt $\mathbf{B}$ to produce intermediate $\mathbf{C}$. The loss of the second dimethylamine molecule in this step yields the desired product. For the formation of 1,4-oxazpine salt 3a, intermediate D is formed by the intramolecular nucleophilic attack of phenolic oxygen on the obtained iminium salt $\mathbf{B}$. The loss of the dimethylamine molecule form intermediate $\mathbf{D}$, yields $\mathbf{3 a}$.

\section{Experimental}

All chemicals were purchased from Merck or Fluka chemical companies. The ${ }^{1} \mathrm{H}$ NMR (300 and $400 \mathrm{MHz}$ ) and ${ }^{13} \mathrm{C}$ NMR spectra (75 and $100 \mathrm{MHz}$ ) were run on a Bruker Avance 400 . Tetramethylsilane (TMS) was used as the internal standard for the NMR analysis. IR spectra were recorded using an FTIR apparatus. Melting points were recorded on a Stuart Scientific Apparatus SMP3 (UK) in open capillary tubes. Elemental C, H and $\mathrm{N}$ analyses, were performed using a Costech CHNS-O 
Table 2 Synthesis of product 3 via the reaction of 2 -substituted trimethinium salts 1 with aminophenol derivatives 2 in the presence of Et ${ }_{3} \mathrm{~N}$ in ethanol at reflux

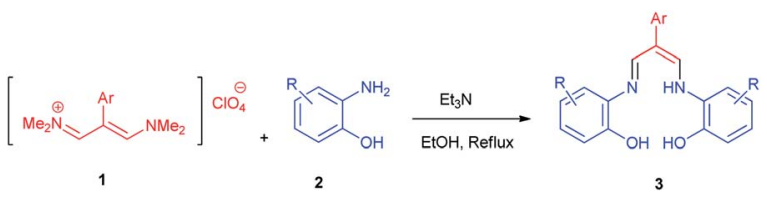

\begin{tabular}{llllll}
\hline Entry & Trimethinium salts $\mathbf{1}$ & $\mathrm{R}$ & Product $\mathbf{3}^{\text {Time }(\mathrm{h})}$ & Yield $^{a}(\%)$ \\
\hline
\end{tabular}

1

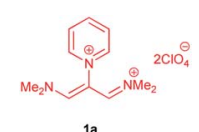

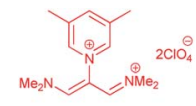

16

$1 \mathbf{b}$

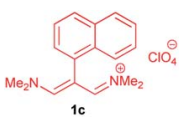

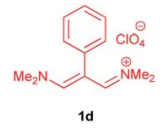

1d

6

7

8

4

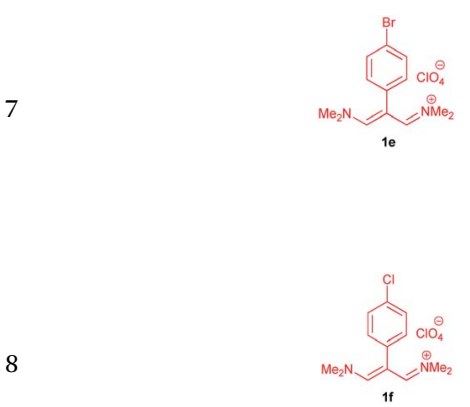

$\mathrm{H}$

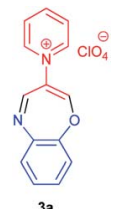

за

H
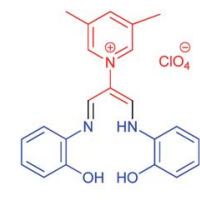

3b

(P)

$\mathrm{CH}_{3}$<smiles>COc1cccc(C)c1NCc1ccccc1-c1cccc(C)c1</smiles>

10

Cl

$\mathrm{CH}_{3}$

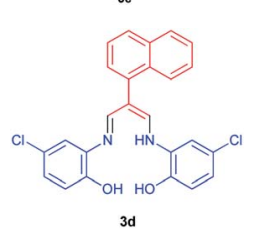

15

12

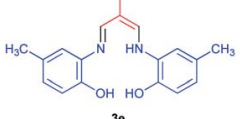

Cl

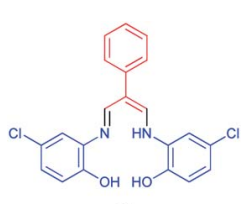

14

Cl

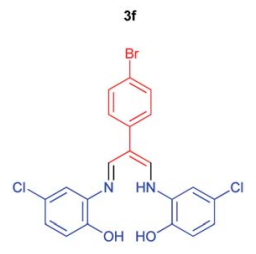

12

Cl

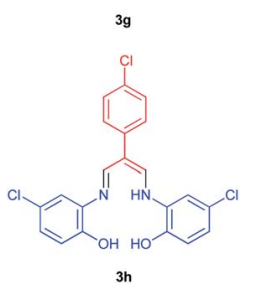

98

88

60

90

95

90

88

0

90

93 
Table 2 (Contd.)

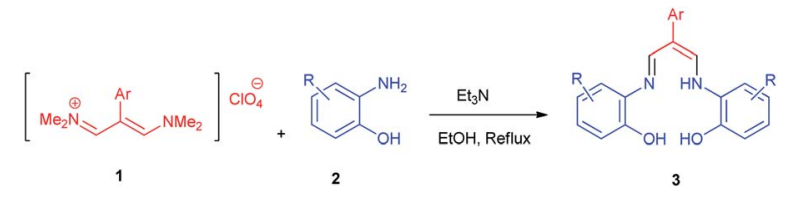

\begin{tabular}{llll}
\hline Entry & Trimethinium salts 1 & $\mathrm{R}$ & Time $^{(\mathrm{h})}$ \\
\hline${ }^{a}$ Isolated yield. & If & Product 3 \\
9 &
\end{tabular}

elemental analyzer. UV/Vis absorption spectra were recorded at room temperature in DMSO using a Perkin-Elmer Lambda 25 spectrophotometer. MS spectra were recorded with a Agilent 7000 Series Triple Quad-MS mass spectrometer.

\section{General procedure for the synthesis of Schiff base derivatives} 3

A mixture of trimethinium salts $1 \mathbf{a}-\mathbf{f}(1 \mathrm{mmol})$ and trimethylamine $(1.0 \mathrm{~mL})$ were dissolved in boiling ethanol $(8 \mathrm{~mL})$.

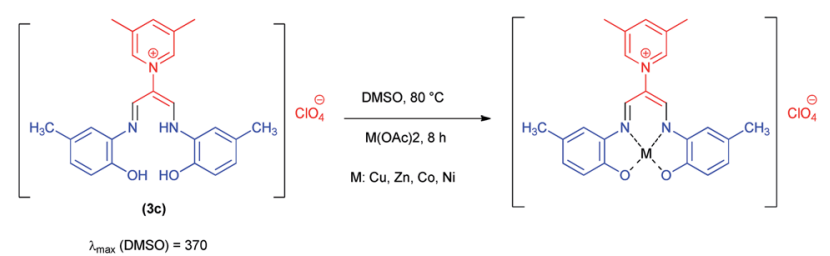

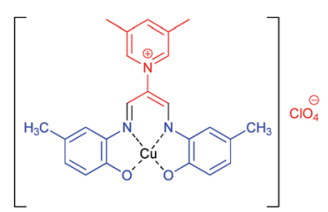

$\lambda_{\max }(\mathrm{DMSO})=412$

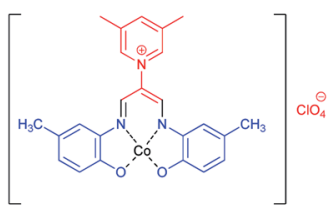

$\lambda_{\max }(\mathrm{DMSO})=415$

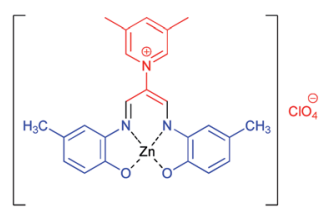

$\lambda_{\max }(\mathrm{DMSO})=400$

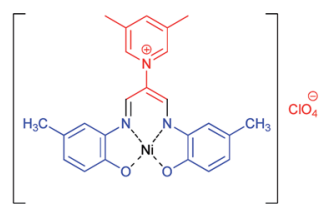

$\lambda_{\max }(D M S O)=416$
Scheme 3 Preparation of Schiff base complexes ${ }^{a}$. ${ }^{a}$ Reaction conditions: 1-((2-hydroxy-5-methylphenyl)amino)-3-(((2-hydroxy-5methylphenyl)imino)prop-1-en-2-yl)-3,5-dimethylpyridinium perchlorate $3 \mathrm{c}(1 \mathrm{mmol}), \mathrm{M}(\mathrm{OAc})_{2}(\mathrm{M} \sim \mathrm{Cu}, \mathrm{Zn} \mathrm{Co}, \mathrm{Ni})(1 \mathrm{mmol}), \mathrm{DMSO}$ $(3 \mathrm{~mL})$ at $100^{\circ} \mathrm{C}$ for $6 \mathrm{~h}$.
Aminophenols 2a-c ( $2 \mathrm{mmol})$ in EtOH $(7 \mathrm{~mL})$ were added dropwise to the stirred mixture at reflux ethanol for $12 \mathrm{~h}$. (2-Amino-4chlorophenol and 2-amino-4-nitrophenol in ethanol $(7 \mathrm{~mL})$ and other aminophenols in methanol $(7 \mathrm{~mL})$ was dissolved). After completion of the reaction confirmed by TLC, the reaction mixture was set aside in a refrigerator for $12 \mathrm{~h}$. Then the solvent

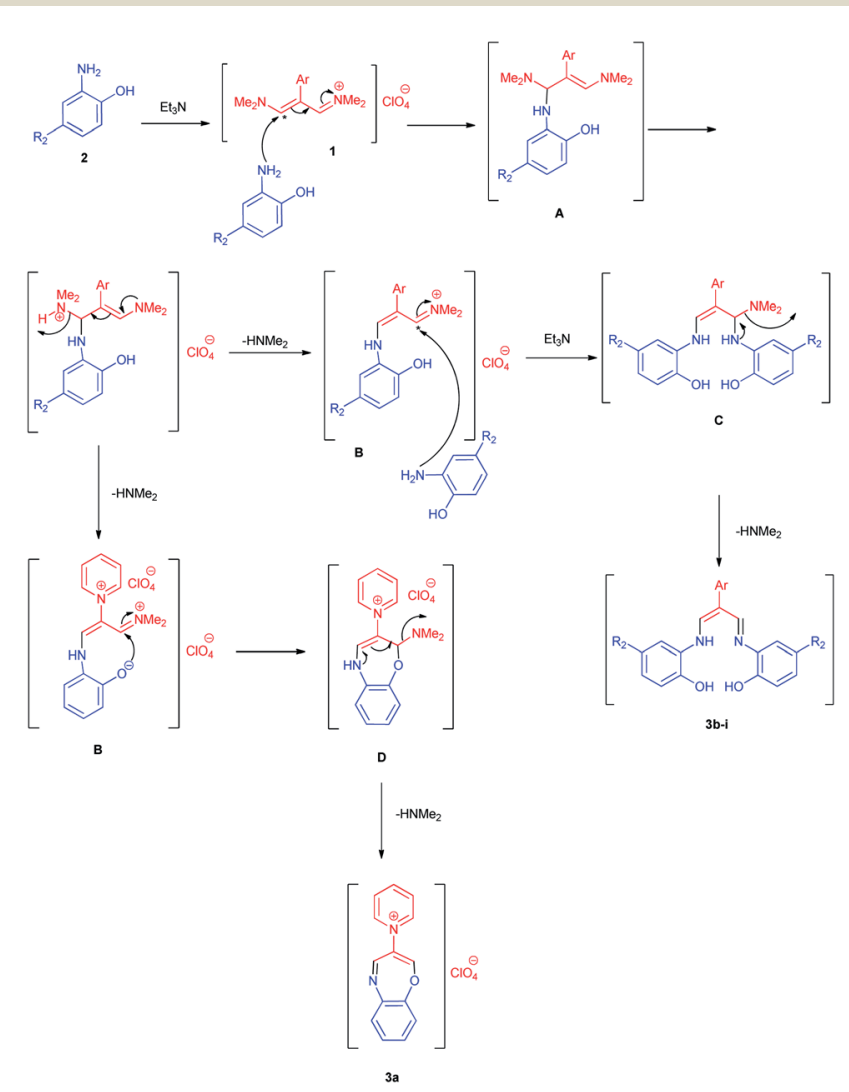

Scheme 4 Proposed mechanism for the synthesis product $3 a-i$. 
was evaporated under vacuum, and a small amount of dichloromethane $(10 \mathrm{~mL})$ was added, and the precipitate formed was collected by filtration, recrystallized from 2-propanol, and dried in vacuum at $80{ }^{\circ} \mathrm{C}$ to afford the pure product $3 \mathbf{a}-\mathbf{i}$.

\section{General procedure for the synthesis metal complexes of Schiff} base $3 c$

A solution of Schiff base $3 \mathbf{c}(1 \mathrm{mmol})$ and acetate salt of transition metals copper(II), zinc(II), cobalt(II) or nickel(II) $(1 \mathrm{mmol})$ in DMSO $(3 \mathrm{~mL})$ was heated at $100{ }^{\circ} \mathrm{C}$ for $6 \mathrm{~h}$. After completion of the reaction confirmed by TLC, the reaction mixture was cooled to room temperature, then a small amount of water (3 $\mathrm{mL}$ ) was added and the precipitate formed was collected by filtration, recrystallized from distilled water $(5 \mathrm{~mL})$ to get pure products in excellent yield.

\section{1-(Benzo $[b][1,4]$ oxazepin-3-yl)pyridinium perchlorate 3a}

Red powder, mp $>260{ }^{\circ} \mathrm{C},{ }^{1} \mathrm{H}$ NMR $\left(\right.$ DMSO- $\left._{6}, 400 \mathrm{MHz}\right)$ $\delta(\mathrm{ppm}): 6.79(\mathrm{~d}, J=6.4 \mathrm{~Hz}, 2 \mathrm{H}), 6.88-6.95(\mathrm{~m}, 2 \mathrm{H}), 7.56(\mathrm{~s}, 1 \mathrm{H})$, $8.24(\mathrm{t}, J=7.2 \mathrm{~Hz}, 2 \mathrm{H}), 8.27(\mathrm{~s}, 1 \mathrm{H}), 8.72(\mathrm{t}, J=7.8 \mathrm{~Hz}, 1 \mathrm{H}), 9.11$ $(\mathrm{d}, J=5.6 \mathrm{~Hz}, 2 \mathrm{H}) .{ }^{13} \mathrm{C}$ NMR (DMSO- $\left.d_{6}, 100 \mathrm{MHz}\right) \delta(\mathrm{ppm})$ : $114.3,115.8,119.7,119.9,125.8,128.0,138.8,146.9,147.8$, 149.2, 150.4, 156.0. Anal. calcd for $\left(\mathrm{C}_{14} \mathrm{H}_{11} \mathrm{~N}_{2} \mathrm{O}\right)\left(\mathrm{ClO}_{4}\right): \mathrm{C}, 52.11$; $\mathrm{H}, 3.44 ; \mathrm{N}, 8.68 \%$. Found: $\mathrm{C}, 52.13 ; \mathrm{H}, 3.45 ; \mathrm{N}, 8.67 \%$. $\lambda_{\max }$ $(\mathrm{DMSO})=340 \mathrm{~nm}$.

1-((2-Hydroxyphenyl)amino)-3-(((2-hydroxyphenyl)imino) propen-2-yl)-3,5-dimethylpyridinium perchlorate $3 \mathrm{~b}$

Red powder, mp $>260{ }^{\circ} \mathrm{C},{ }^{1} \mathrm{H}$ NMR $\left(D_{M S O}-d_{6}, 300 \mathrm{MHz}\right.$ ) $\delta(\mathrm{ppm}): 2.52(\mathrm{~s}, 6 \mathrm{H}), 3.62-4.52(\operatorname{broad}, 3 \mathrm{H}), 6.72(\mathrm{t}, J=7.2 \mathrm{~Hz}$, $2 \mathrm{H}), 6.82(\mathrm{~d}, J=8.0 \mathrm{~Hz}, 4 \mathrm{H}), 6.93(\mathrm{t}, J=7.4 \mathrm{~Hz}, 2 \mathrm{H}), 8.28(\mathrm{~s}, 2 \mathrm{H})$, $8.63(\mathrm{~s}, 1 \mathrm{H}), 8.99(\mathrm{~s}, 2 \mathrm{H}) .{ }^{13} \mathrm{C}$ NMR (DMSO- $\left.d_{6}, 100 \mathrm{MHz}\right) \delta(\mathrm{ppm})$ : 18.2, 116.8, 117.8, 118.2, 126.1, 133.0, 138.1, 142.2, 145.3, 145.8, 147.5, 152.1. Anal. calcd for $\left(\mathrm{C}_{22} \mathrm{H}_{22} \mathrm{~N}_{3} \mathrm{O}_{2}\right)\left(\mathrm{ClO}_{4}\right): \mathrm{C}, 57.46 ; \mathrm{H}$, 4.82; N, 9.14\%. Found: C, 57.44; H, 4.83; N, 9.15\%. IR (KBr) $\left(\nu_{\max }, \mathrm{cm}^{-1}\right): 3508,3365,1645,1099 \mathrm{~cm}^{-1} \cdot \lambda_{\max }(\mathrm{DMSO})=$ $355 \mathrm{~nm}$.

1-((2-Hydroxy-5-methylphenyl)amino)-3-(((2-hydroxy-5methylphenyl)imino)propen-2-yl)-3,5-dimethylpyridinium perchlorate $3 \mathrm{c}$

Orang powder, $\mathrm{mp}>260{ }^{\circ} \mathrm{C},{ }^{1} \mathrm{H}$ NMR (DMSO- $d_{6}, 300 \mathrm{MHz}$ ) $\delta(\mathrm{ppm}): 2.27$ (s, 6H), 2.54 (s, 6H), 6.78-6.85 (m, 4H), $7.27(\mathrm{~s}, 2 \mathrm{H})$, $8.37(\mathrm{~d}, 1 \mathrm{H}), 8.60(\mathrm{~s}, 2 \mathrm{H}), 9.01(\mathrm{~s}, 2 \mathrm{H}), 9.39(\mathrm{broad}, 2 \mathrm{H}), 12.34(\mathrm{~s}$, $1 \mathrm{H}) .{ }^{13} \mathrm{C}$ NMR (DMSO- $\left.d_{6}, 75 \mathrm{MHz}\right) \delta$ (ppm): 18.2, 20.9, 116.0, 118.2, 118.8, 126.5, 129.1, 132.4, 138.2, 142.6, 145.8, 146.5, 147.0. Anal. calcd for $\left(\mathrm{C}_{24} \mathrm{H}_{26} \mathrm{~N}_{3} \mathrm{O}_{2}\right)\left(\mathrm{ClO}_{4}\right): \mathrm{C}, 59.08 ; \mathrm{H}, 5.37$; , 8.61\%. Found: C, 59.10; H, 5.39; N, 8.60\%. IR (KBr) $\left(\nu_{\max }, \mathrm{cm}^{-1}\right)$ : $3500,1645,1375,1090 \mathrm{~cm}^{-1}$. MS $m / z$ calcd for $\mathrm{C}_{24} \mathrm{H}_{26} \mathrm{~N}_{3} \mathrm{O}_{2}[\mathrm{M}]^{+}$ 388.4, found 388.2. $\lambda_{\max }(\mathrm{DMSO})=370 \mathrm{~nm}$.

4-Chloro-3-((5-chloro-2-hydroxyphenyl)amino)-2(((naphthalen-1-yl)allylidene)amino)phenol 3d

Yellow powder, mp $>260{ }^{\circ} \mathrm{C},{ }^{1} \mathrm{H}$ NMR $\left(\mathrm{DMSO}-d_{6}, 300 \mathrm{MHz}\right)$ $\delta$ (ppm): 6.87 (d, $J=8.4 \mathrm{~Hz}, 2 \mathrm{H}), 7.09(\mathrm{dd}, J=1.6,8.5 \mathrm{~Hz}, 1 \mathrm{H})$, $7.43(\mathrm{~s}, 1 \mathrm{H}), 7.59-7.73(\mathrm{~m}, 5 \mathrm{H}), 7.84(\mathrm{~m}, 2 \mathrm{H}), 8.09-8.15(\mathrm{~m}, 2 \mathrm{H})$, $8.35(\mathrm{~s}, 1 \mathrm{H}), 8.74(\mathrm{~s}, 1 \mathrm{H}), 10.36$ (broad, $1 \mathrm{H}) .{ }^{13} \mathrm{C}$ NMR (DMSO- $d_{6}$, $75 \mathrm{MHz}) \delta$ (ppm): 106.9, 117.8, 119.3, 123.5, 125.0, 126.4, 126.6, 127.3, 128.0, 128.1, 128.3, 129.3, 130.3, 130.4, 132.3, 134.0, 147.1, 159.2, 164.7. Anal. calcd for $\mathrm{C}_{25} \mathrm{H}_{18} \mathrm{Cl}_{2} \mathrm{~N}_{2} \mathrm{O}_{2}$ : C, 66.83; $\mathrm{H}$, 4.04; N, 6.23\%. Found: C, 66.85; H, 4.05; N, 6.21\%. MS $m / z$ calcd for $\mathrm{C}_{25} \mathrm{H}_{18} \mathrm{Cl}_{2} \mathrm{~N}_{2} \mathrm{O}_{2}[\mathrm{M}]^{+} 449.3$, found 449. $\lambda_{\max }(\mathrm{DMSO})=$ $340 \mathrm{~nm}$.

3-((2-Hydroxy-5-methylphenyl)amino)-2-((phenylallylidene) amino)-4-methylphenol $3 \mathrm{e}$

Yellow powder, $\mathrm{mp}>260{ }^{\circ} \mathrm{C},{ }^{1} \mathrm{H}$ NMR (DMSO- $d_{6}, 300 \mathrm{MHz}$ ) $\delta$ (ppm): 2.27 (s, 6H), 6.88-6.95 (m, 4H), 7.27 (s, 2H), 7.49-7.68 $(\mathrm{m}, 5 \mathrm{H}), 8.79(\mathrm{~s}, 1 \mathrm{H}), 9.61-9.62(\mathrm{~m}, 1 \mathrm{H}), 10.21(\mathrm{~s}, 1 \mathrm{H}) .{ }^{13} \mathrm{C} \mathrm{NMR}$ (DMSO- $\left.d_{6}, 75 \mathrm{MHz}\right) \delta$ (ppm): 20.7, 111.8, 116.6, 120.4, 126.3, 128.4, 129.0, 129.2, 129.9, 130.6, 130.7, 140.5, 158.6. Anal. calcd for $\mathrm{C}_{23} \mathrm{H}_{22} \mathrm{~N}_{2} \mathrm{O}_{2}$ : C, 77.07; H, 6.19; N, 7.82\%. Found: C, 77.06; H, $6.21 ; \mathrm{N}, 7.80 \%$. $\lambda_{\max }(\mathrm{DMSO})=335 \mathrm{~nm}$.

\section{4-Chloro-3-((5-chloro-2-hydroxyphenyl)amino)-2- ((phenylallylidene)amino)phenol 3f}

Brown powder, $\mathrm{mp}>260{ }^{\circ} \mathrm{C},{ }^{1} \mathrm{H}$ NMR $\left(\mathrm{DMSO}-d_{6}, 400 \mathrm{MHz}\right.$ ) $\delta(\mathrm{ppm}): 6.86(\mathrm{t}, J=7.4 \mathrm{~Hz}, 2 \mathrm{H}), 6.92-6.99(\mathrm{~m}, 3 \mathrm{H}), 7.42(\mathrm{~d}, J=$ $7.6 \mathrm{~Hz}, 2 \mathrm{H}), 7.46$ (d, $J=7.6 \mathrm{~Hz}, 2 \mathrm{H}), 7.53$ (s, 2H), 8.39 (d, $J=$ $6 \mathrm{~Hz}, 2 \mathrm{H}$ ), 9.39 (broad, 2H), 12.85 (s, 1H). ${ }^{13} \mathrm{C}$ NMR (DMSO- $d_{6}$, $100 \mathrm{MHz}) \delta$ (ppm): 111.7, 115.0, 122.5, 127.5, 127.7, 128.0, 129.1, 129.5, 129.7, 130.5, 140.6, 152.6. Anal. calcd for $\mathrm{C}_{21} \mathrm{H}_{16} \mathrm{Cl}_{2} \mathrm{~N}_{2} \mathrm{O}_{2}$ : C, 63.17; H, 4.04; N, 7.02\%. Found: C, 63.15; H, 4.05; N, 7.01\%. $\lambda_{\max }(\mathrm{DMSO})=330 \mathrm{~nm}$.

\section{2-(4-Bromophenyl)-3-((((5-chloro-2-hydroxyphenyl)amino)} allylidene)amino)-4-chlorophenol $3 g$

Brown powder, $\mathrm{mp}>260{ }^{\circ} \mathrm{C},{ }^{1} \mathrm{H}$ NMR (DMSO- $d_{6}, 300 \mathrm{MHz}$ ) $\delta(\mathrm{ppm}): 6.90(\mathrm{~d}, J=8.4 \mathrm{~Hz}, 2 \mathrm{H}), 6.97(\mathrm{dd}, J=2.2,8.5 \mathrm{~Hz}, 2 \mathrm{H})$, $7.52-7.59(\mathrm{~m}, 6 \mathrm{H}), 8.41$ (d, $J=5.7 \mathrm{~Hz}, 2 \mathrm{H}), 9.82$ (broad, $2 \mathrm{H})$, $12.82(\mathrm{t}, J=5.8 \mathrm{~Hz}, 1 \mathrm{H}) .{ }^{13} \mathrm{C}$ NMR (DMSO- $\left.d_{6}, 75 \mathrm{MHz}\right) \delta(\mathrm{ppm})$ : $108.9,117.1,117.4,118.6,124.0,124.1,128.4,131.7,135.2$, 139.3, 147.2, 149.8. Anal. calcd for $\mathrm{C}_{21} \mathrm{H}_{15} \mathrm{BrCl}_{2} \mathrm{~N}_{2} \mathrm{O}_{2}$ : C, 52.75; H, 3.16; N, 5.86\%. Found: C, 52.78; H, 3.15; N, 5.84\%. $\lambda_{\max }$ $(\mathrm{DMSO})=360 \mathrm{~nm}$.

2-(4-Chlorophenyl)-3-((((5-chloro-2-hydroxyphenyl)amino) allylidene)amino)-4-chlorophenol $3 \mathrm{~h}$

Brown powder, $\mathrm{mp}>260{ }^{\circ} \mathrm{C},{ }^{1} \mathrm{H}$ NMR (DMSO- $d_{6}, 400 \mathrm{MHz}$ ) $\delta(\mathrm{ppm}): 6.91(\mathrm{~d}, J=8.4 \mathrm{~Hz}, 2 \mathrm{H}), 6.97(\mathrm{~d}, J=8.4 \mathrm{~Hz}, 2 \mathrm{H}), 7.38(\mathrm{~s}$, $2 \mathrm{H}), 7.58(\mathrm{~d}, J=12.4 \mathrm{~Hz}, 2 \mathrm{H}), 7.70(\mathrm{~d}, J=12 \mathrm{~Hz}, 2 \mathrm{H}), 8.45(\mathrm{~d}, J=$ $4.8 \mathrm{~Hz}, 2 \mathrm{H}), 9.89$ (broad, $2 \mathrm{H}), 12.82$ (s, $1 \mathrm{H}) .{ }^{13} \mathrm{C}$ NMR (DMSO- $d_{6}$, $100 \mathrm{MHz}) \delta$ (ppm): 110.0, 117.1, 117.4, 123.8, 123.9, 126.6, 132.7, 133.8, 135.3, 137.0, 147.4, 149.6. Anal. calcd for $\mathrm{C}_{21} \mathrm{H}_{15} \mathrm{Cl}_{3} \mathrm{~N}_{2} \mathrm{O}_{2}$ : C, 58.15; H, 3.49; N, 6.46\%. Found: C, 58.14; H, 3.47; N, 6.47\%. $\lambda_{\text {max }}(\mathrm{DMSO})=345 \mathrm{~nm}$. 
2-(4-Chlorophenyl)-3-((((2-hydroxyphenyl)amino)allylidene) amino)phenol $3 \mathbf{i}$

Brown powder, $\mathrm{mp}>260{ }^{\circ} \mathrm{C},{ }^{1} \mathrm{H}$ NMR (DMSO- $d_{6}, 400 \mathrm{MHz}$ ) $\delta(\mathrm{ppm}): 6.78-6.89(\mathrm{~m}, 8 \mathrm{H}), 7.00(\mathrm{~d}, J=7.2 \mathrm{~Hz}, 2 \mathrm{H}), 7.11(\mathrm{~d}, J=$ $7.6 \mathrm{~Hz}, 2 \mathrm{H}), 8.31$ (d, $J=5.6 \mathrm{~Hz}, 2 \mathrm{H}), 8.99$ (broad, 2H), 12.00 (s, 1H). ${ }^{13} \mathrm{C}$ NMR (DMSO- $\left.d_{6}, 100 \mathrm{MHz}\right) \delta(\mathrm{ppm}): 115.3,120.8,124.7$, 126.0, 127.5, 131.3, 133.9, 140.0, 141.3, 150.3, 158.4, 162.2. Anal. calcd for $\mathrm{C}_{21} \mathrm{H}_{17} \mathrm{ClN}_{2} \mathrm{O}_{2}$ : C, 69.14; H, 4.70; N, 7.68\%. Found: C, $69.15 ; \mathrm{H}, 4.72 ; \mathrm{N}, 7.67 \%$. $\lambda_{\max }(\mathrm{DMSO})=350 \mathrm{~nm}$.

\section{Conclusions}

In conclusion, we have reported a highly efficient method for the synthesis of important Schiff base derivatives via a condensation reaction between 2 -substituted trimethinium salts as starting compounds with aminophenol in presence of triethylamine in ethanol at reflux. A simple procedure in the excellent yields, mild reaction conditions, and metal-catalyst free are the main advantages of this method. Metal complexes of Schiff base can be used as catalysts and to advance a number of reactions such as carbon-carbon or carbon-nitrogen coupling reactions, which are separately planned for future works.

\section{Conflicts of interest}

There are no conflicts to declare.

\section{Acknowledgements}

The authors thank Persian Gulf University Research Councils for the financial support of this work.

\section{Notes and references}

1 C. M. da Silva, D. L. da Silva, L. V. Modolo, R. B. Alves, M. A. de Resende, C. V. B. Martins and A. de Fatima, Schiff bases: A short review of their antimicrobial activities, $J$. Adv. Res., 2011, 2, 1, DOI: 10.1016/j.jare.2010.05.004.

2 S. Kumar, D. N. Dhar and P. N. Saxena, Application of metal complexes of Schiff bases - A review, J. Sci. Ind. Res., 2009, 68, 181. http://nopr.niscair.res.in/handle/123456789/3170.

3 P. Przybylski, A. Huczynski, K. Pyta, B. Brzezinski and F. Bartl, Biological properties of Schiff bases and azo derivatives of phenols, Curr. Org. Chem., 2009, 13, 124, DOI: $10.2174 / 138527209787193774$.

4 Z. Guo, R. Xing, S. Liu, Z. Zhong, X. Ji, L. Wang, et al., Antifungal properties of Schiff bases of chitosan, Nsubstituted chitosan and quaternized chitosan, Carbohydr. Res., 2007, 342, 1329, DOI: 10.1016/j.carres.2007.04.006.

5 A. Carreño, C. Zúñiga, D. Páez-Hernández, M. Gacitúa, R. Polanco, C. Otero, R. Arratia-Pérez and J. A. Fuentes, Study of structure-bioactivity relationship of three new pyridine Schiff bases: Synthesis, spectral characterization, DFT calculations and biological assays, New J. Chem., 2018, 42, 8851, DOI: 10.1039/c8nj00390d.
6 Z. D. Petrović, J. Đorović, D. Simijonović, V. P. Petrović and Z. Marković, Experimental and theoretical study of antioxidative properties of some salicylaldehyde and vanillic Schiff bases, $R S C A d v$, 2015, 5, 24094, DOI: 10.1039/c5ra02134k.

7 P. G. Cozzi, Metal-Salen Schiff base complexes in catalysis: practical aspects, Chem. Soc. Rev., 2004, 33, 410, DOI: 10.1039/b307853c.

$8 \mathrm{H}$. Schiff, Mittheilungen aus dem Universitätslaboratorium in Pisa: Eine neue Reihe organischer Basen, Justus Liebigs Ann. Chem., 1864, 131, 118, DOI: 10.1002/jlac.18641310113.

9 T. P. Yoon and E. N. Jacobsen, Privileged Chiral Catalysts, Science, 2003, 299, 1691, DOI: 10.1126/science.1083622.

10 R. H. Holm, G. W. Everett Jr and A. Chakravorty, Metal Complexes of Schiff Bases and $\beta$-Ketoamines, Prog. Inorg. Chem., 1966, 7, 85, DOI: 10.1002/9780470166086.ch3.

11 A. Hasaninejad, S. Mojikhalifeh and M. Beyrati, Highly efficient, catalyst-free, one-pot, pseudo five-component synthesis of novel pyrazoline-containing Schiff bases, metal complexes formation and computational studies via DFT method, Appl. Organomet. Chem., 2018, 32, e4380, DOI: $10.1002 /$ aoc. 4380 .

12 M. Tripathi, V. Regnier, Z. Ziani, M. Devillard, C. Philouze and D. Martin, Metal free oxidation of vinamidine derivatives: a simple synthesis of $\alpha$-keto- $\beta$-diimine ligands, RSC Adv., 2018, 8, 38346, DOI: 10.1039/c8ra08220k.

13 D. L. Ostercamp, Y. Dinh, D. Graff and S. Wiles, Rigid Core Vinamidinium Salts and Their $\mathrm{N}, \mathrm{N}^{\prime}$-Rotamers, J. Org. Chem., 2003, 68, 3099, DOI: 10.1021/jo020654l.

14 F. Azamifar, M. R. Naimi-Jamal, A. Rineh and M. J. Kelso, Synthesis, structural/photophysical characterization and theoretical investigations with new $\beta$-pyridinium/ quinolinium and $\beta$-bromine substituted bis(1,3dimethylbarbituric acid)trimethine oxonol dyes that display large Stokes shifts, Dyes Pigm., 2020, 172, 107758, DOI: 10.1016/j.dyepig.2019.107758.

15 H. Yamamaka, H. Takekawa, K. Morita, T. Ishihara and J. T. Gupton, Preparation of novel $\beta$-trifluoromethyl vinamidinium salt and its synthetic application to trifluoromethylated heterocycles, Tetrahedron Lett., 1996, 37, 1829, DOI: 10.1016/0040-4039(96)00129-3.

16 J. F. Marcoux, F. A. Marcotte, J. Wu, P. G. Dormer, I. W. Davies, D. Hughes and P. J. Reider, A general preparation of pyridines and pyridones via the annulation of ketones and esters, J. Org. Chem., 2001, 66, 4194, DOI: 10.1021/jo0155198.

17 C. Reichardt and K. Halbritter, Synthesen mit substituierten Malondialdehyden, $\quad$ X. $\gamma$-Fluor-pentamethincyaninFarbstoffe, Chem. Ber., 1971, 104, 822, DOI: 10.1002/ cber.19711040319.

18 D. Lloyd and H. McNab, Vinamidines and Vinamidinium Salts-Examples of Stabilized Push-Pull Alkenes, Angew. Chem., Int. Ed. Engl., 1976, 15, 459, DOI: 10.1002/ anie.197604591.

19 R. T. Clemens and S. Q. Smith, The application of vinamidinium salts to the synthesis of 2,4-disubstituted 
thiophenes, Tetrahedron Lett., 2005, 46, 1319, DOI: 10.1016/ j.tetlet.2004.12.113.

20 Z. Arnold, Synthetic reactions of dimethylformamide. XII. Formylation of some carboxylic acids and their derivatives, Collect. Czech. Chem. Commun., 1961, 26, 3051, DOI: 10.1135/cccc19613051.

21 F. Azamifara, M. R. Naimi-Jamal and O. M. Demchuk, Design, synthesis and characterization of new trimethine oxonol dyes from 1,3-indandione and 2-substituted vinamidinium salts, Dyes Pigm., 2019, 161, 438, DOI: 10.1016/j.dyepig.2018.09.043.

22 J. Hu, J. Wang, R. Liu, Y. Li, J. Lu and H. Zhu, Aggregationinduced-emission-active vinamidinium salts with tunable emissions, reversible mechanochromic response and the application in data-security protection, Dyes Pigm., 2018, 153, 84, DOI: 10.1016/j.dyepig.2018.02.007.

23 E. Szennyes, G. Gyémánt, L. Somsák and É. Bokor, Synthesis of New Series of 2-C-( $\beta$-D-glucopyranosyl)-Pyrimidines and Their Evaluation as Inhibitors of Some Glycoenzymes, Molecules, 2020, 25, 701, DOI: 10.3390/molecules25030701.

24 A. M. Mehranpour, S. Hashemnia and R. Maghamifar, Synthesis and Characterization of New $\gamma$-Substituted Pentamethine Cyanine Dyes, Synth. Commun., 2010, 40, 3594, DOI: 10.1080/00397910903457290.

25 A. M. Mehranpour, S. Hashemnia and Z. Shayan, Synthesis and characterization of new derivatives of 1,4-diazepinium salts, Synth. Commun., 2011, 41, 3501, DOI: 10.1080/ 00397911.2010.518332.
26 N. Golzar, A. M. Mehranpour and N. Nowrouzi, A facile and efficient route to one-pot synthesis of new cyclophanes using vinamidinium salts, $R S C A d v$. , 2021, 11, 13666, DOI: 10.1039/ d0ra10548a.

27 Z. Rafiee Samani, A. M. Mehranpour and A. Hasaninejad, Preparation of 2,5-disubstituted pyrimidines from vinamidinium salts and synthesis of novel disulfane derivatives, J. Heterocycl. Chem., 2020, 57, 2150, DOI: 10.1002/jhet.3935.

28 F. Habashi, A. M. Mehranpour and E. B. Jahromi, Synthesis and characterization of new derivatives of bis(1,4-diazepinium) salts and $\operatorname{bis}(\gamma$-substituted pentamethine cyanine) dyes using vinamidinium salt, $J$. Heterocycl. Chem., 2020, 57, 2428, DOI: 10.1002/jhet.3958.

29 Z. Rafiee Samani and A. M. Mehranpour, Synthesis of novel 5 -substituted isophthalates from vinamidinium salts, Tetrahedron Lett., 2019, 60, 151002, DOI: 10.1016/ j.tetlet.2019.151002.

30 A. M. Mehranpour and M. Zahiri, Synthesis and characterization of new benzimidazole derivatives using 2substituted 1,3-bis(dimethylamino)-trimethinium salts, Tetrahedron Lett., 2014, 55, 3969, DOI: 10.1016/ j.tetlet.2014.05.038.

31 A. M. Mehranpour, S. Hashemnia and F. Azamifar, Synthesis of new malonaldehyde derivatives using 2-heteroarylsubstituted trimethinium salts, Tetrahedron Lett., 2013, 54, 321, DOI: 10.1016/j.tetlet.2012.11.046. 